\title{
Plasma proteomic profiling reveals biomarkers associated with aortic dilation in patients with bicuspid aortic valve
}

\author{
Wenrui $\mathrm{Ma}^{1}$, Jingjing Zhang ${ }^{2}$, Kehua $\mathrm{Xu}^{2}$, Shiqiang Yan ${ }^{2}$, Dingqian Liu ${ }^{1}$, Hui Huang ${ }^{2}$, Yuyi Tang ${ }^{2}$, \\ Guoquan Yan ${ }^{2}$, Yongxin Sun ${ }^{1}$, Jun Li ${ }^{1}$, Weijia Zhang ${ }^{2}$, Chunsheng Wang ${ }^{1}$, Kai Zhu ${ }^{1}$, Hao Lai ${ }^{1}$ \\ ${ }^{1}$ Department of Cardiac Surgery and Shanghai Institute of Cardiovascular Diseases, Zhongshan Hospital, Fudan University, Shanghai, China; \\ ${ }^{2}$ Institutes of Biomedical Sciences and Shanghai Key Laboratory of Medical Imaging Computing and Computer-Assisted Intervention, Shanghai \\ Medical College, Fudan University, Shanghai, China \\ Contributions: (I) Conception and design: W Zhang, K Zhu, C Wang, H Lai; (II) Administrative support: W Zhang, K Zhu, C Wang, H Lai; (III) \\ Provision of study materials or patients: Y Sun, J Li, K Zhu, C Wang, H Lai; (IV) Collection and assembly of data: W Ma, J Zhang, K Xu, S Yan, D \\ Liu, H Huang, Y Tang, G Yan, Y Sun, J Li; (V) Data analysis and interpretation: W Ma, J Zhang, K Xu, S Yan; (VI) Manuscript writing: All authors; \\ (VII) Final approval of manuscript: All authors. \\ Correspondence to: Kai Zhu, MD, PhD; Chunsheng Wang, MD; Hao Lai, MD. Department of Cardiac Surgery and Shanghai Institute of \\ Cardiovascular Diseases, Zhongshan Hospital, Fudan University, 180 Fenglin Road, Shanghai 200032, China. Email: zhu.kai1@zs-hospital.sh.cn; \\ wangchunsheng@fudan.edu.cn; lai.hao@zs-hospital.sh.cn.
}

Background: Bicuspid aortic valve (BAV) is the most common congenital heart anomaly and is prone to cause complications, such as valvular stenosis and thoracic aortic dilation. There is currently no reliable way to predict the progression rate to thoracic aortic aneurysm. Here, we aimed to characterize the proteomic landscape in the plasma of stenotic BAV patients and provide potential biomarkers to predict progressive aortic dilation.

Methods: Plasma samples were obtained from 45 subjects (30 stenotic BAV patients and 15 healthy controls). All samples were properly prepared and analyzed using mass spectrometry (MS)-based label-free quantitative proteomics.

Results: A total of 748 plasma proteins had missingness $<50 \%$, and $193(25.8 \%)$ were differentially expressed in the BAV patients. Functions regarding cell junction and actin cytoskeleton were largely enriched. NOTCH3, a Notch receptor known to interact with the BAV-causing gene NOTCH1, was negatively correlated with aortic diameter and was downregulated in BAV patients' plasma and aortic smooth muscle cells. Further, a subset of plasma proteins, including ADAM10, was associated with rapidly progressive aortic dilation in BAV patients.

Conclusions: Our data reveal unique features in the proteomic architecture of stenotic BAV patients' plasma, and we propose the potential of Notch signaling proteins NOTCH3 and ADAM1O in predicting aortic dilation.

Keywords: Plasma proteomics; bicuspid aortic valve (BAV); aortic dilation; biomarker; Notch signaling

Submitted May 18, 2021. Accepted for publication Jul 13, 2021.

doi: $10.21037 / \mathrm{atm}-21-3378$

View this article at: https://dx.doi.org/10.21037/atm-21-3378

\section{Introduction}

Bicuspid aortic valve (BAV) is the most frequent congenital heart anomaly affecting approximately $1.4 \%$ of the general population (1-3). Ascending aortic disease is a common complication of a stenotic BAV, characterized by progressive aortic dilation (i.e., an increase in the aortic diameter) that may lead to aneurysm formation and lethal events such as aortic dissection and rupture (4-6). Currently, there is no 
validated method to predict the progression rate of aortic dilation in BAV patients, thus posing an obstacle to clinical decision-making regarding the optimal timing for aortic surgery $(7,8)$. Plasma proteins have emerged as a useful source of biomarkers to reflect aortic pathology at the tissue level $(9,10)$ but have not been comprehensively profiled in BAV patients with aortic dilation.

In this study, we collected plasma samples from stenotic BAV patients and healthy controls. By mass spectrometry (MS)-based proteomic analysis, the entire list of the differentially expressed proteins (DEPs) was identified and characterized. Further, the ascending aortic diameters of the BAV patients were measured during follow-up, and the DEPs were screened for potential biomarkers that were closely related to progressive aortic dilation. We present the following article in accordance with the MDAR reporting checklist (available at https://dx.doi.org/10.21037/atm-21-3378).

\section{Methods}

\section{Study subjects and materials}

In this study, we included adult patients with stenotic BAV who were hospitalized at our institution between December 2017 and November 2018. Exclusion criteria included patients with connective tissue disorder, predominant aortic valve regurgitation, or a history of cardiac surgery. After screening, a total of 30 eligible candidates were enrolled, including 17 patients with significant aortic dilation (ascending aortic diameter $>45 \mathrm{~mm}$ ) and 13 without (diameter $\leq 45 \mathrm{~mm}$ ). We further recruited 15 healthy volunteers without cardiovascular diseases (ascending aortic diameter $\leq 35 \mathrm{~mm}$ ) as the control group. To evaluate the plasma proteins' capacity to reflect pathologies in the aortic cells, we harvested and expanded primary aortic smooth muscle cells from an additional three BAV patients with significant aortic dilation and from two controls with non-diseased ascending aortas, using the aortic explant technique (11). For all cell experiments, the passage number was limited to six. This study was approved by Zhongshan Hospital Fudan University Ethics Committee (Approval Letter No. B2020-158R), and written consent was obtained from all study subjects. All procedures performed in this study involving human participants were in accordance with the Declaration of Helsinki (as revised in 2013).

\section{Sample preparation for MS analysis}

Plasma samples were collected from all patients and volunteers and depleted using the High Select ${ }^{\mathrm{TM}}$ Top14 Abundant Protein Depletion mini column (Thermo Fisher Scientific). After measuring the plasma protein concentration (Pierce BCA, Thermo Fisher Scientific), $50 \mu \mathrm{g}$ protein for each sample were reduced with $10 \mathrm{mM}$ dithiothreitol (final concentration) for 30 minutes at $37^{\circ} \mathrm{C}$ and alkylated with $55 \mathrm{mM}$ iodoacetamide (final concentration) for an additional 30 minutes at room temperature. The remaining detergent was removed by acetone precipitation, and protein pellets were resolved in $50 \mu \mathrm{L} 6 \mathrm{M}$ urea (in $10 \mathrm{mM}$ Hepes, $\mathrm{pH}$ 8.0) and digested with $0.5 \mu \mathrm{g} \mathrm{LysC}$ for 3 hours at room temperature. After adding four volumes of $50 \mathrm{mM}$ ammonium bicarbonate, tryptic digestion was carried out overnight. On the next day, digestion was stopped by adding $1 \%$ trifluoroacetic acid. Peptides were finally desalted on C18 Stage Tips and kept at $-80{ }^{\circ} \mathrm{C}$ until MS analysis.

\section{Liquid chromatography-MS analysis}

MS analysis was performed using a Quadrupole Orbitrap mass spectrometer (Q Exactive HF, Thermo Fisher Scientific) coupled to a Waters uHPLC system via a nano-electrospray source. Columns were packed with $1.9 \mathrm{~mm} \mathrm{C18}$ particles (Thermo Fisher Scientific). Peptides were separated over a 250 -minute gradient from $2 \%$ to $60 \%$ in buffer B ( $80 \%$ acetonitrile, $0.5 \%$ formic acid) at $200 \mathrm{~nL} /$ minute. The column temperature was constantly set to $50{ }^{\circ} \mathrm{C}$ using a column oven. The survey scans were acquired with a resolution of 60,000 for Q Exactive HF, at $\mathrm{m} / \mathrm{z} 200$. A top 15 method was used to select the most abundant precursor ions with a charge $Z^{2+}$. Selected precursor ions were subjected to high-energy collisional dissociation fragmentation at a normalized collision energy of 27 , an isolation window of $1.4 \mathrm{Th}$, and a resolution of 15,000 at $\mathrm{m} / \mathrm{z} 200$. For survey scans, ion injection times were set to 20 milliseconds (target value $3 \mathrm{E} 6$ ) and 120 milliseconds (target value 1E5) for MS/MS scans. Dynamic exclusion of sequenced peptides was set to 30 seconds. Data were acquired using Xcalibur software (Thermo Fisher Scientific).

\section{MS data analysis}

MS raw files were analyzed using MaxQuant software (Computational Systems Biochemistry). Proteins with missing values $<50 \%$ were filled using $\mathrm{k}$ Nearest Neighbors followed by normalization using the edge R R package 
(version 3.28.1) (12,13). The limma $\mathrm{R}$ package (version 3.42.2) was used to examine whether proteins were differentially expressed between BAV and control groups and between $\mathrm{BAV}$ patients with and without progressive aortic dilation (14). Proteins with $\mathrm{P}$ values $<0.05$, and foldchange ratios $>1.5$ were considered significant. Annotation and functional enrichment were performed using the Metascape database (http://metascape.org) and the Ingenuity Pathway Analysis (IPA, QIAGEN), and proteinprotein interactions were searched using the STRING database (15-18). The comparative proteomics datasets are uploaded online (https://cdn.amegroups.cn/static/public/ atm-21-3378-1.xlsx). The raw MS data files are available from the corresponding authors upon reasonable request.

\section{Criterion for progressive aortic dilation}

In this study, 17 stenotic BAV patients with an ascending aortic diameter $\geq 45 \mathrm{~mm}$ underwent aortic valve replacement and concomitant aortic repair, and 12 out of 13 patients with an ascending aortic diameter $<45 \mathrm{~mm}$ underwent isolated valve replacement. Among these patients, nine were followed up at our institution after surgery. Given the potential interobserver variability in measuring aortic diameter, progressive aortic dilation was defined conservatively, i.e., a gradual increase in the ascending aortic diameter over time that reached $\geq 4 \mathrm{~mm}$ at the latest follow-up.

\section{Quantitative real-time polymerase chain reaction (qRT-PCR)}

The total RNA of the primary aortic smooth muscle cells expanded from BAV patients and controls were extracted using TRIzol agent (T9424, Sigma-Aldrich). Synthesis of complementary DNA and qRT-PCR was performed according to the manufacturer's guidelines (RR037B and RR420B, Takara). The expression levels of the following genes were investigated: $A D A M 10$ (forward: ATGGGAGGTCAGTATGGGAATC; reverse: ACTGCTCTTTTGGCACGCT), HEY2 (forward: AAGGCGTCGGGATCGGATAA; reverse: AGAGCGTGTGCGTCAAAGTAG), NOTCH3 (forward: TGGCGACCTCACTTACGACT; reverse: CACTGGCAGTTATAGGTGTTGAC), SERPINE1 (forward: ACCGCAACGTGGTTTTCTCA; reverse: TTGAATCCCATAGCTGCTTGAAT), SUMO 4 (forward: CCACGGGGATTGTCAGTGAAG; reverse: CTGTGCAGGTTTGTCTGTTCC), SVEP1 (forward: CAGCAGTTGCATTCCATGTCC; reverse: AAGTAACCATTTTCGGGAGGC), and GAPDH (forward: CTGGGCTACACTGAGCACC; reverse: AAGTGGTCGTTGAGGGCAATG).

\section{Immunofluorescence}

Immunofluorescence was performed on the diseased and control aortic smooth muscle cells. Briefly, cells were fixed in $4 \%$ paraformaldehyde at room temperature for 20 minutes and permeabilized with $0.1 \%$ Triton-X (X100, Sigma-Aldrich) solution at room temperature for 30 minutes. NOTCH3 and smooth muscle cell marker ACTA2 were stained using their respective primary antibodies (D11B8, Cell Signaling Technology; ab7817, Abcam) overnight at $4{ }^{\circ} \mathrm{C}$. After three washes with PBS, the samples were incubated with secondary antibodies (4412S and 8890S, Cell Signaling Technology) for 1 hour at $37{ }^{\circ} \mathrm{C}$. Nuclei were stained with 4',6-diamidino-2phenylindole (DAPI, D1306, Invitrogen) for 5 minutes at room temperature. Representative images of three different regions of each sample were acquired using a fluorescence microscope (DMi8, Leica) and analyzed using ImageJ software (ver. 1.52n, National Institutes of Health).

\section{Enzyme-linked immunosorbent assay (ELISA)}

The concentration of ADAM10 in the supernatant of the BAV and control aortic smooth muscle cells was measured using a sandwich ELISA kit (JL13337, Jianglai Biology, Shanghai, China) that contained adhered anti-human ADAM10 antibodies. Secondary antibodies were used to bind to the adhered proteins and, after adding substrate to the enzyme, the absorbance reading of the plates was performed on a plate reader at $450 \mathrm{~nm}$ wavelength (AMR100, Allsheng, Hangzhou, China).

\section{Statistical analysis}

Continuous variables were presented as means \pm standard deviations or median (interquartile range), according to the normality test. Parameters were compared using the two-tailed $t$-test (normal distribution) or the MannWhitney U-test (skewed distribution). Categorical variables were expressed as numbers (percentages) and compared using Fisher's exact test or the Cochran-Mantel-Haenszel test, as appropriate. Correlation analysis was performed 
using Pearson's correlation test. Analyses were performed using Prism v8.0 (GraphPad Software, Inc.). Statistical significance was indicated by two-tailed ${ }^{*} \mathrm{P}<0.05,{ }^{*} \mathrm{P}<0.01$, and ${ }^{* * *} \mathrm{P}<0.001$.

\section{Results}

\section{Characteristics of the study population and the overall proteome}

The study workflow is shown in Figure $1 A$, and the baseline demographics of the study subjects are listed in Table S1 and Table S2. There was no significant difference in age, gender, or other clinical parameters between the BAV patients and healthy controls. Using the plasma samples harvested from all candidates, proteomic analysis identified a total of 998 proteins, 748 of which had missingness $<50 \%$. Annotation showed the top 20 functions of these plasma proteins, including aortic disease-related functions such as insulin-like growth factor transport, extracellular matrix organization, and cell-substrate adhesion (Figure 1B). The principal component analysis suggested distinct proteomic profiles between BAV patients and healthy controls with marginal overlaying regions, while stratification by gender or age did not clearly distinguish the overall plasma proteome, suggesting the presence of independent features in the plasma of stenotic BAV patients (Figure 1C).

\section{Analysis of DEPs between BAV patients and controls}

The comparative analysis yielded a total of 193 DEPs (Figure 2A; Table S3). Disease enrichment analysis revealed cardiovascular diseases such as vascular disease and abdominal aortic aneurysm (Figure S1). The integrated process enrichment analysis using Metascape showed a network of enriched terms, including regulated exocytosis, platelet degranulation, extracellular structure organization, and others (Figure S2). The canonical pathway enrichment analysis using IPA software indicated that the DEPs were related to epithelial adherens junction, RhoA signaling, integrin signaling, and actin cytoskeleton signaling (Figure $2 B$ ). The top upstream regulators included ADAM metallopeptidase domain 10 (ADAM10), matrix metallopeptidase 12 (MMP12), transforming growth factor beta 1 (TGFB1), and GATA binding protein 4 (GATA4), all of which are associated with thoracic aortic aneurysm (Figure 2C) (19-21). Notably, ADAM10 is a regulator of the Notch signaling pathway that causes BAV formation
(22-24). Since components and concentrations of plasma proteins could be influenced by alterations in the whole human body, 16 DEPs that are involved in several aortic diseases were screened (Figure 2D), including collagen type I alpha 1 chain (COL1A1), fibrillin-1 (FBN1), filamin A (FLNA), and notch receptor 3 (NOTCH3), which were closely interacted in a protein network (Figure $2 E)(5,25)$. Expression-trait correlation analysis showed that $\mathrm{NOTCH} 3$ was independently associated with the ascending aortic diameter. Expressions of COL1A1 and FBN1 were poorly correlated with aortic size, and FLNA expression was significantly influenced by the patient's age (Figure $2 F$ ).

\section{NOTCH3 was consistently downregulated in the plasma and aortic cells of $B A V$ patients}

The Notch receptors have an overlapping effect on the development of multiple cardiovascular diseases (26). Mutation of the NOTCH1 gene is a known causative factor of BAV and thoracic aortic aneurysm (24), whereas the role of NOTCH3 in such diseases remains unclear. The correlation between the plasma level of NOTCH3 and the ascending aortic diameter was in a negative direction (Figure $3 A$ ). Analysis of the protein-protein interactions indicated a close relationship across NOTCH1, NOTCH3, and ADAM10 (Figure 3B). Further, qRT-PCR showed downregulation of $\mathrm{NOTCH} 3$ and its downstream effector [hes related family bHLH transcription factor with YRPW motif 2 (HEY2)] in the aortic smooth muscle cells of BAV patients (Figure 3C). The immunofluorescence analysis also showed reduced levels of NOTCH3 and ACTA2 in diseased cells (Figure 3D).

\section{DEPs between patients with and without progressive aortic dilation}

The follow-up measurement of ascending aortic diameters is shown in Figure 4A. Progressive aortic dilation that reached the surgical threshold was present in two patients (4). The baseline characteristics of patients with and without progressive aortic dilation were comparable. Comparative proteomic analysis identified a total of 74 DEPs (Figure 4B; Table S4). When compared with the DEPs between BAV patients and controls, 23 proteins were overlapped. Notably, we identified 11 proteins exhibiting consistent changing trends in both comparisons (Figure 4C). Top-hit proteins such as ADAM10, plasminogen activator inhibitor 1 (SERPINE1), small ubiquitin-like 

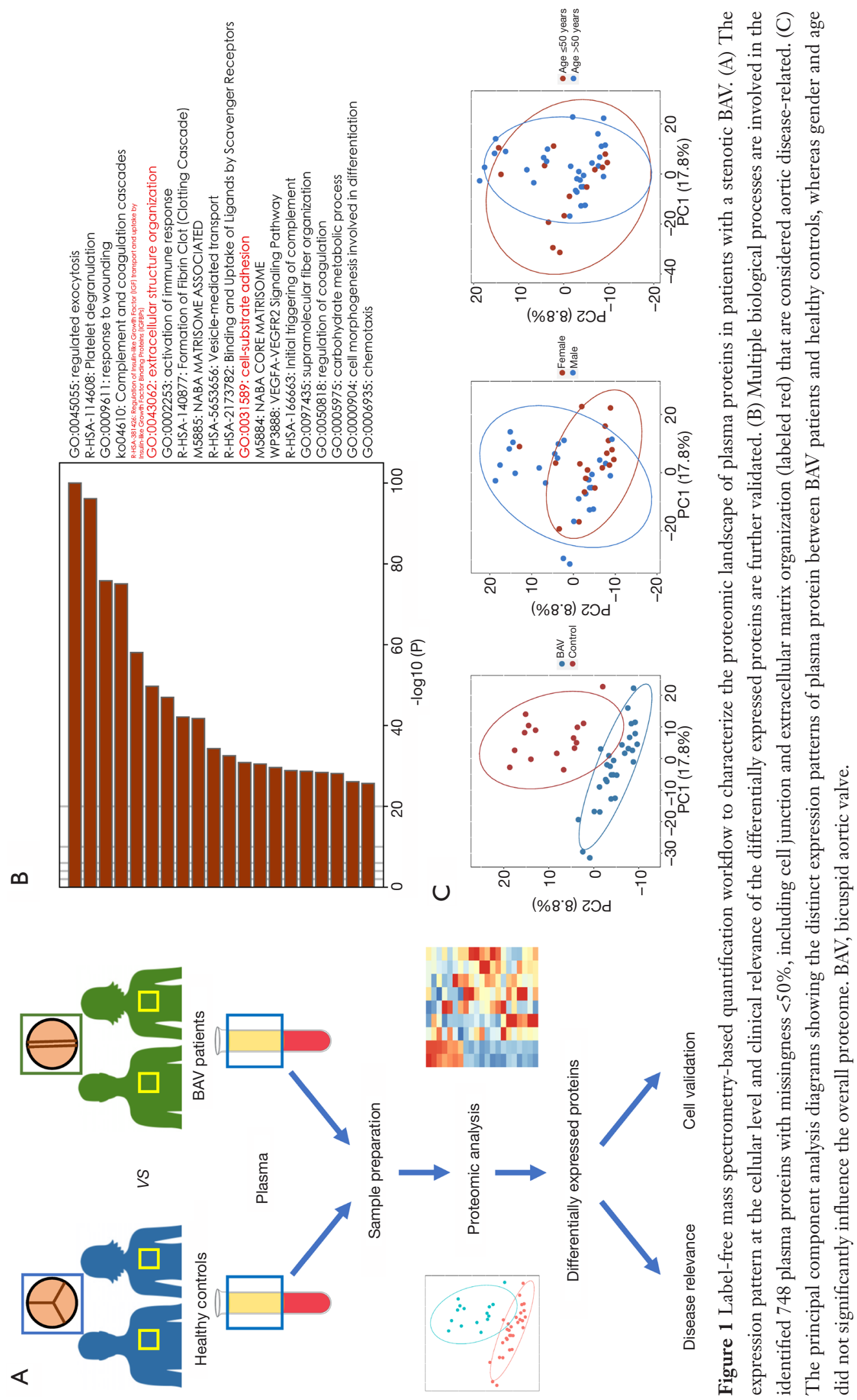

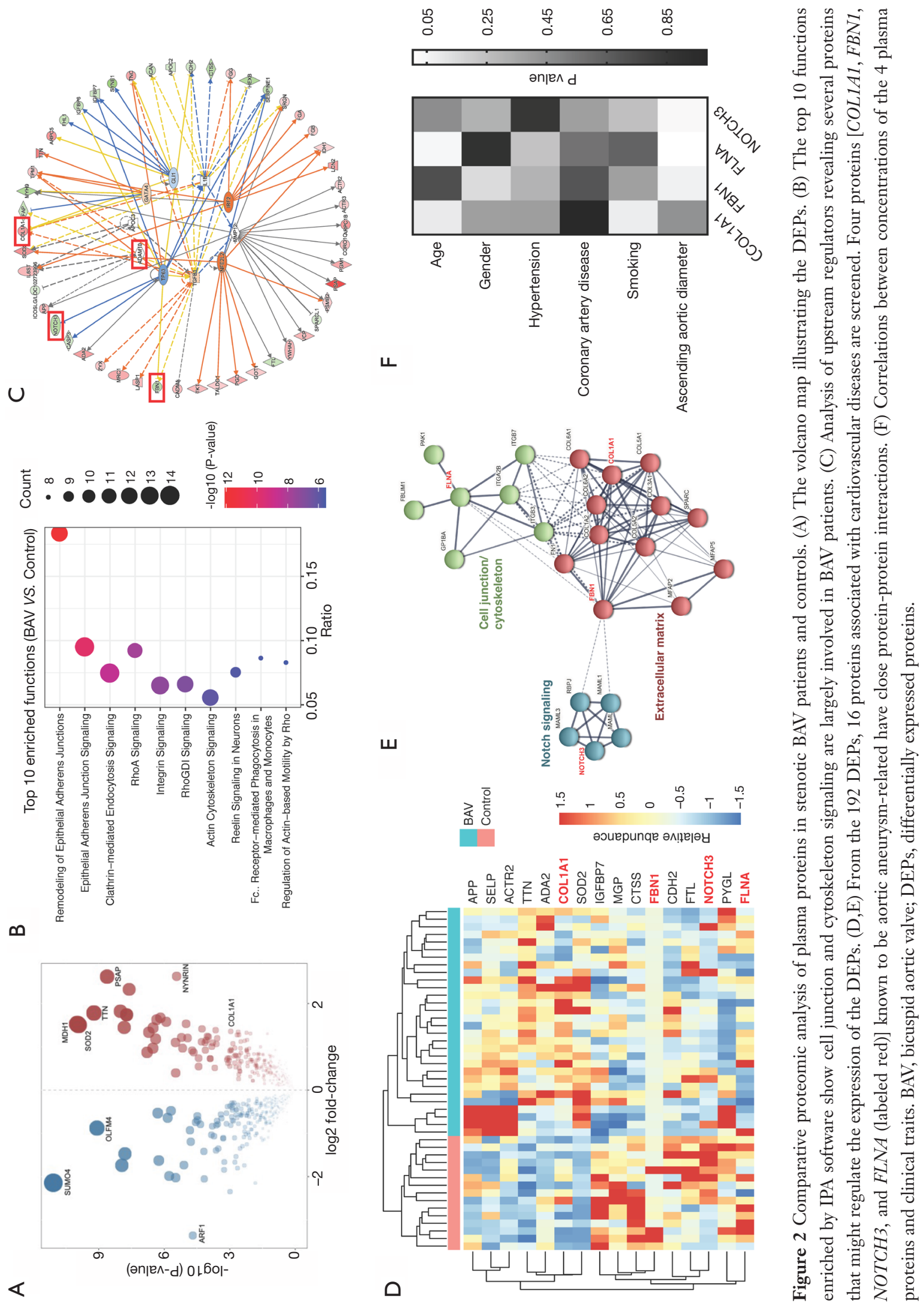

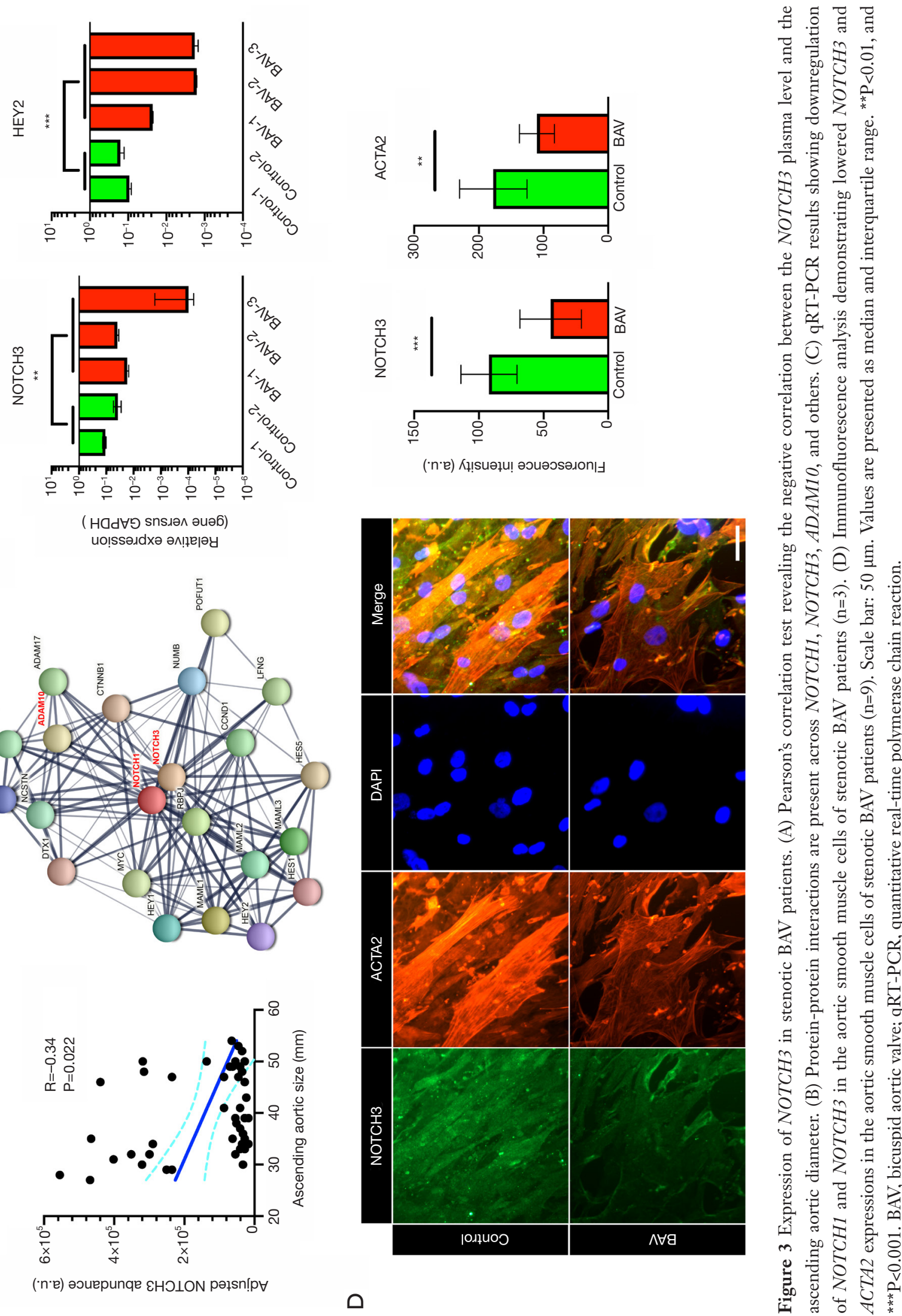

$\infty$

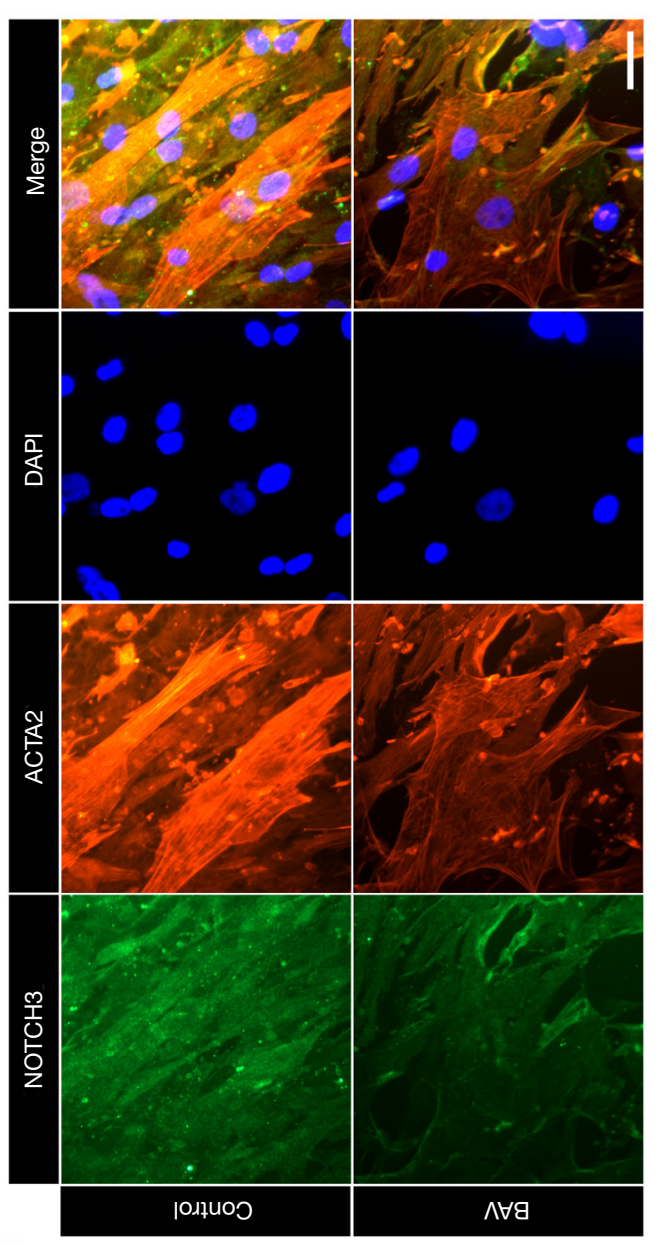

( HOdHD snsıәл әиә6)

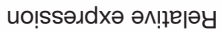

$\cup$
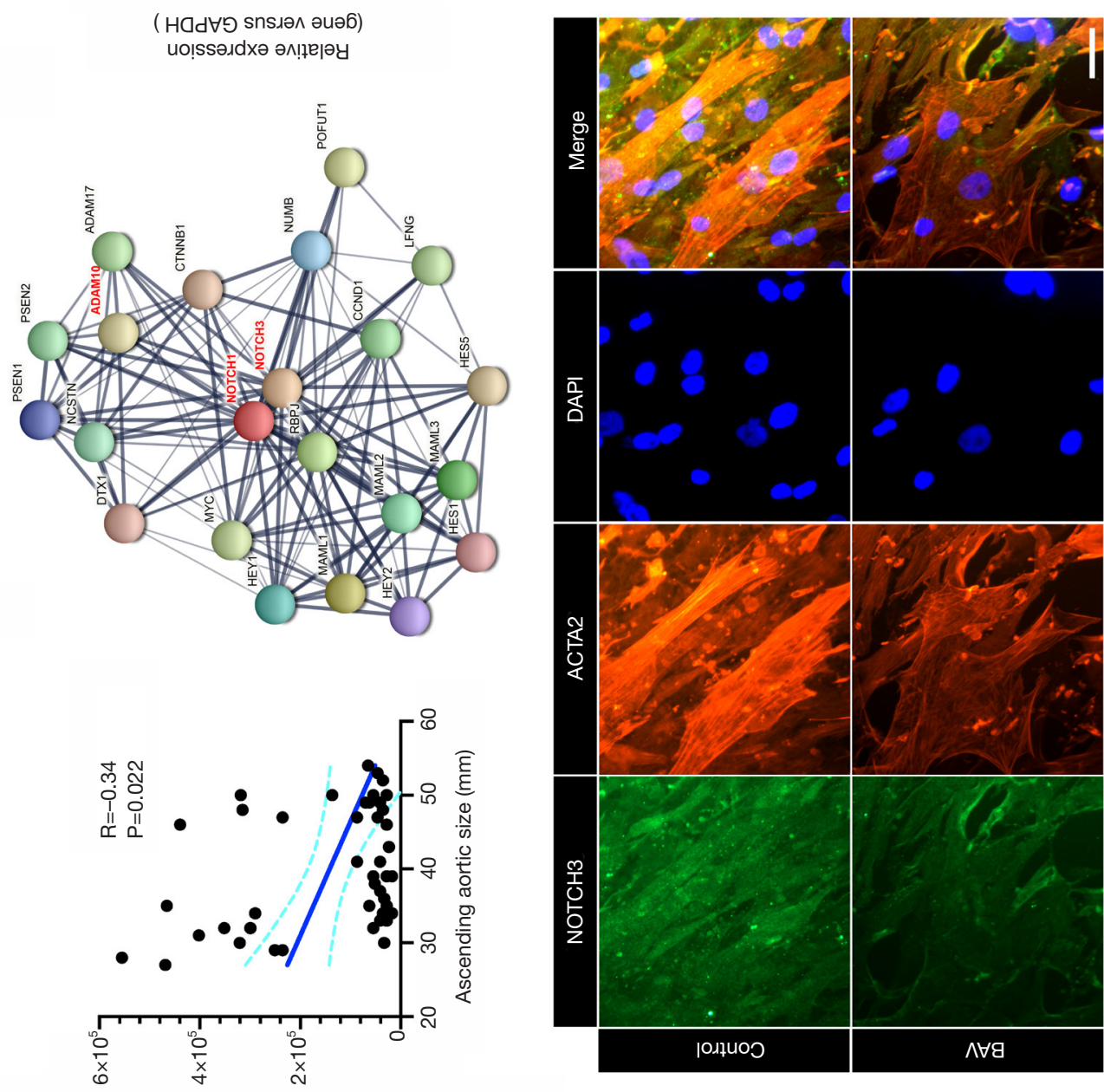

$\varangle$

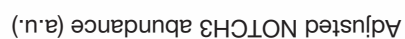

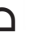



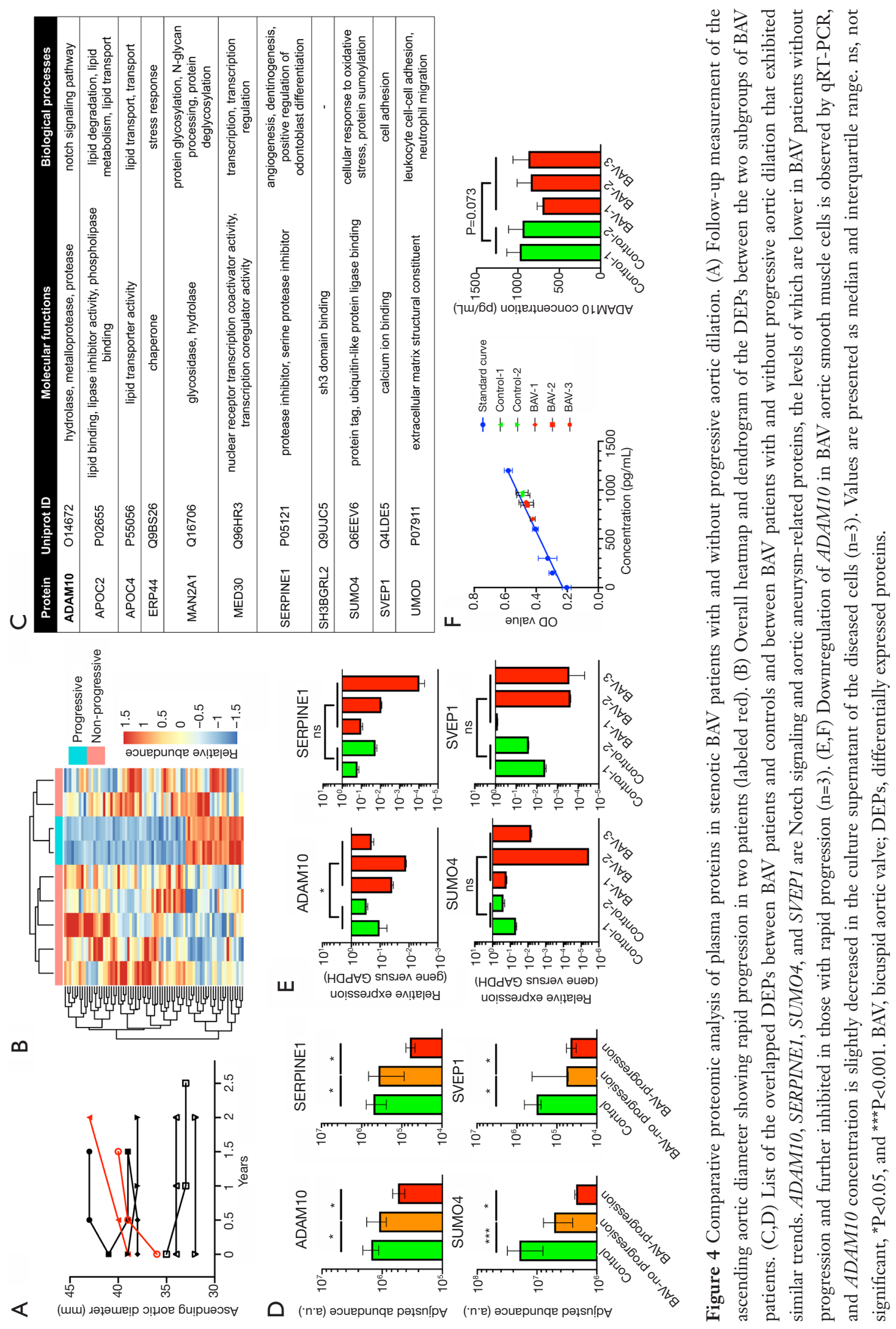
modifier 4 (SUMO4), and sushi, von Willebrand factor type A, EGF and pentraxin domain containing 1 (SVEP1), were downregulated in BAV patients and further inhibited in those with progressive aortic dilation (Figure $4 D$ ). Transcription of the $A D A M 10$ gene was consistently downregulated in BAV aortic smooth muscle cells, while other proteins did not show a similar trend (Figure 4E). The concentration of $A D A M 10$ was higher in the supernatant of control cells, although the difference did not quite reach the threshold of statistical significance $(\mathrm{P}=0.073$; Figure $4 F)$.

\section{Discussion}

BAV-related aortic dilation is a complicated disease associated with genetic mutations and aberrant hemodynamics (1). To date, the molecular mechanism of aortic disease in patients with BAV remains unclear. In this study, proteomic analysis showed that patients with a stenotic BAV had plasma protein profiles distinct from those of controls. Proteins abnormally expressed in thoracic aortic aneurysm tissues exhibited similar alterations in the plasma, indicating the potential of plasma biomarkers in predicting tissue pathologies. For instance, NOTCH3 was relevant to the existing degree of aortic dilation (a static state), while the plasma level of $A D A M 10$ indicated rapidly progressive aortic dilation (a dynamic state). Our findings shed light on the distinct characteristics in the plasma proteome of BAV patients, which could be exploited to identify biomarkers that could predict disease progression or outcomes.

From a general perspective, the proteomic landscape of BAV patients' plasma differed significantly from that of healthy controls, which corresponded with the fact that aortic dilation in BAV patients may commence early in life, suggesting an intrinsic defect in this population (3). The identified DEPs between BAV patients and controls included several thoracic aortic aneurysm-related proteins, namely COL1A1, FBN1, FLNA, and NOTCH3. It is well established that mutations in COL1A1, FBN1, and FLNA cause familial thoracic aortic aneurysm (20,27-29), but in this study, the plasma levels of these proteins were not independently associated with the ascending aortic diameter. The Notch receptor family member NOTCH1 is associated with $\mathrm{BAV}$ as well as thoracic aortic aneurysm $(2,24)$. As an important interactor of NOTCH1, NOTCH3 is also reported to promote protective remodeling pathways in vascular smooth muscle cells $(24,30)$. In this study, we observed an independent correlation between the NOTCH3 level and the ascending aortic diameter, and downregulation of $\mathrm{NOTCH}_{3}$ and the Notch downstream effector HEY2 in the diseased aortic smooth muscle cells, implying that systemic NOTCH3 insufficiency in stenotic BAV patients might be traced to pathologies in the aorta.

Rapid progressive ascending aortic dilation commonly results in a higher risk of morbidity and mortality than stable dilation (4). Considering the high prevalence of $\mathrm{BAV}$ in the general population, the biological difference between patients with and without progressive aortic dilation is clinically meaningful and should be clarified. In this study, a subset of proteins was correlated with progressive aortic dilation. ADAM10 is a metalloprotease that controls the proteolytic processing of Notch receptors: Whilst insufficiency in expression of Notch receptors results in lower levels of the Notch intracellular domain in the cytoplasm and transcriptional activation of Notch target genes, downregulation of ADAM10 may block the liberation of the Notch intracellular domain, further impairing Notch signaling (22). Consistently, we observed downregulation of $A D A M 10$ in aortic smooth muscle cells. Other top-hit proteins, namely SERPINE1, SUMO4, and SVEP1, were also associated with progressive aortic dilation but were not evidently downregulated in aortic smooth muscle cells. This discrepancy could be explained by the fact that the plasma protein network arises partly via systemic cross-tissue regulations. A follow-up validation of these primary data is warranted to adjust for bias derived from other tissues and organs (31). Moreover, the type and degree of valvular dysfunction may also contribute to the pattern of aortic dilation (1). Future studies may also include more patients with multiple valve configurations to provide a comprehensive landscape of BAV plasma proteomics. Although our study only included patients with stenotic BAV, this proteomics method can be applied to other causes of thoracic aortic aneurysm, namely sporadic (tricuspid) and Marfan cases.

In conclusion, the current study is the first to profile plasma proteomics in patients with a stenotic BAV. The potential for plasma proteins such as NOTCH3 and $A D A M 10$ to predict aortic dilation is also proposed, which merits further mechanistic and clinical investigations.

\section{Acknowledgments}

The authors thank Hangqi Shen, MD, PhD, Shanghai Jiao Tong University, for her support in manuscript preparation. 
Funding: This work was supported by grants from the National Natural Science Foundation of China (81771971, 82070482, 81772007, and 82070484), the Science and Technology Commission of Shanghai Municipality (20ZR1411700), and the Shanghai Municipal Science and Technology Major Project (2017SHZDZX01).

\section{Footnote}

Reporting Checklist: The authors have completed the MDAR reporting checklist. Available at https://dx.doi. org/10.21037/atm-21-3378

Data Sharing Statement: Available at https://dx.doi. org/10.21037/atm-21-3378

Conflicts of Interest: All authors have completed the ICMJE uniform disclosure form (available at https://dx.doi. org/10.21037/atm-21-3378). The authors have no conflicts of interest to declare.

Ethical Statement: The authors are accountable for all aspects of the work in ensuring that questions related to the accuracy or integrity of any part of the work are appropriately investigated and resolved. All procedures performed in this study involving human participants were in accordance with the Declaration of Helsinki (as revised in 2013). This study was approved by Zhongshan Hospital Fudan University Ethics Committee (Approval Letter No. B2020-158R), and written consent was obtained from all study subjects.

Open Access Statement: This is an Open Access article distributed in accordance with the Creative Commons Attribution-NonCommercial-NoDerivs 4.0 International License (CC BY-NC-ND 4.0), which permits the noncommercial replication and distribution of the article with the strict proviso that no changes or edits are made and the original work is properly cited (including links to both the formal publication through the relevant DOI and the license). See: https://creativecommons.org/licenses/by-nc-nd/4.0/.

\section{References}

1. Verma S, Siu SC. Aortic dilatation in patients with bicuspid aortic valve. N Engl J Med 2014;370:1920-9.

2. Bob-Manuel T, Heckle MR, Ifedili IA, et al. Outcomes of transcatheter aortic valve replacement in bicuspid aortic valve stenosis. Ann Transl Med 2019;7:102.

3. Grattan M, Prince A, Rumman RK, et al. Predictors of Bicuspid Aortic Valve-Associated Aortopathy in Childhood: A Report From the MIBAVA Consortium. Circ Cardiovasc Imaging 2020;13:e009717.

4. Borger MA, Fedak PWM, Stephens EH, et al. The American Association for Thoracic Surgery consensus guidelines on bicuspid aortic valve-related aortopathy: Full online-only version. J Thorac Cardiovasc Surg 2018;156:e41-74.

5. Lo Presti F, Guzzardi DG, Bancone C, et al. The science of BAV aortopathy. Prog Cardiovasc Dis 2020;63:465-74.

6. Pasipoularides A. Clinical-pathological correlations of BAV and the attendant thoracic aortopathies. Part 1: Pluridisciplinary perspective on their hemodynamics and morphomechanics. J Mol Cell Cardiol 2019;133:223-32.

7. Abdulkareem N, Soppa G, Jones S, et al. Dilatation of the remaining aorta after aortic valve or aortic root replacement in patients with bicuspid aortic valve: a 5 -year follow-up. Ann Thorac Surg 2013;96:43-9.

8. Yasuda H, Nakatani S, Stugaard M, et al. Failure to prevent progressive dilation of ascending aorta by aortic valve replacement in patients with bicuspid aortic valve: comparison with tricuspid aortic valve. Circulation 2003;108 Suppl 1:II291-4.

9. Lee R, Cassimee I, Huang H, et al. Integrated Plasma and Tissue Proteomics Reveals Attractin Release by Intraluminal Thrombus of Abdominal Aortic Aneurysms and Improves Aneurysm Growth Prediction in Humans. Ann Surg 2020. [Epub ahead of print]. doi: 10.1097/ SLA.0000000000004439.

10. Pasta S, Agnese V, Gallo A, et al. Shear Stress and Aortic Strain Associations With Biomarkers of Ascending Thoracic Aortic Aneurysm. Ann Thorac Surg 2020;110:1595-604.

11. Huang J, Cheng L, Li J, et al. Myocardin regulates expression of contractile genes in smooth muscle cells and is required for closure of the ductus arteriosus in mice. $\mathrm{J}$ Clin Invest 2008;118:515-25.

12. McCarthy DJ, Chen Y, Smyth GK. Differential expression analysis of multifactor RNA-Seq experiments with respect to biological variation. Nucleic Acids Res 2012;40:4288-97.

13. Robinson MD, McCarthy DJ, Smyth GK. edgeR: a Bioconductor package for differential expression analysis of digital gene expression data. Bioinformatics 2010;26:139-40.

14. Ritchie ME, Phipson B, Wu D, et al. limma powers 
differential expression analyses for RNA-sequencing and microarray studies. Nucleic Acids Res 2015;43:e47.

15. Zhou Y, Zhou B, Pache L, et al. Metascape provides a biologist-oriented resource for the analysis of systemslevel datasets. Nat Commun 2019;10:1523.

16. Ashburner M, Ball CA, Blake JA, et al. Gene ontology: tool for the unification of biology. The Gene Ontology Consortium. Nat Genet 2000;25:25-9.

17. Gene Ontology Consortium. The Gene Ontology resource: enriching a GOld mine. Nucleic Acids Res 2021;49:D325-34.

18. Szklarczyk D, Gable AL, Lyon D, et al. STRING v11: protein-protein association networks with increased coverage, supporting functional discovery in genomewide experimental datasets. Nucleic Acids Res 2019;47:D607-13.

19. Bossone E, Eagle KA. Epidemiology and management of aortic disease: aortic aneurysms and acute aortic syndromes. Nat Rev Cardiol 2021;18:331-48.

20. Ziganshin BA, Bailey AE, Coons C, et al. Routine Genetic Testing for Thoracic Aortic Aneurysm and Dissection in a Clinical Setting. Ann Thorac Surg 2015;100:1604-11.

21. Elefteriades JA, Pomianowski P. Practical genetics of thoracic aortic aneurysm. Prog Cardiovasc Dis 2013;56:57-67.

22. Alabi RO, Farber G, Blobel CP. Intriguing Roles for Endothelial ADAM10/Notch Signaling in the Development of Organ-Specific Vascular Beds. Physiol Rev 2018;98:2025-61.

23. Koenig SN, LaHaye S, Feller JD, et al. Notch1 haploinsufficiency causes ascending aortic aneurysms in

Cite this article as: Ma W, Zhang J, Xu K, Yan S, Liu D, Huang H, Tang Y, Yan G, Sun Y, Li J, Zhang W, Wang C, Zhu K, Lai H. Plasma proteomic profiling reveals biomarkers associated with aortic dilation in patients with bicuspid aortic valve. Ann Transl Med 2021;9(14):1182. doi: 10.21037/atm-213378 mice. JCI Insight 2017;2:e91353.

24. Garg V, Muth AN, Ransom JF, et al. Mutations in NOTCH1 cause aortic valve disease. Nature 2005;437:270-4.

25. Yassine NM, Shahram JT, Body SC. Pathogenic Mechanisms of Bicuspid Aortic Valve Aortopathy. Front Physiol 2017;8:687.

26. Meester JAN, Verstraeten A, Alaerts M, et al. Overlapping but distinct roles for NOTCH receptors in human cardiovascular disease. Clin Genet 2019;95:85-94.

27. Isselbacher EM, Lino Cardenas CL, Lindsay ME. Hereditary Influence in Thoracic Aortic Aneurysm and Dissection. Circulation 2016;133:2516-28.

28. Crosas-Molist E, Meirelles T, López-Luque J, et al. Vascular smooth muscle cell phenotypic changes in patients with Marfan syndrome. Arterioscler Thromb Vasc Biol 2015;35:960-72.

29. Chen MH, Choudhury S, Hirata M, et al. Thoracic aortic aneurysm in patients with loss of function Filamin A mutations: Clinical characterization, genetics, and recommendations. Am J Med Genet A 2018;176:337-50.

30. Biros E, Walker PJ, Nataatmadja M, et al. Downregulation of transforming growth factor, beta receptor 2 and Notch signaling pathway in human abdominal aortic aneurysm. Atherosclerosis 2012;221:383-6.

31. Emilsson V, Ilkov M, Lamb JR, et al. Co-regulatory networks of human serum proteins link genetics to disease. Science 2018;361:769-73.

(English Language Editor: D. Fitzgerald) 


\section{Supplementary}

Table S1 Baseline characteristics of the included BAV patients and healthy controls

\begin{tabular}{lccc}
\hline Demographics & BAV patients $(n=30)$ & Healthy controls $(\mathrm{n}=15)$ & $0.403^{\mathrm{a}}$ \\
\hline Age (years) & $54.1 \pm 13.1$ & $12(80.0)$ & $0.063^{\mathrm{b}}$ \\
Male gender & $15(50.0)$ & $0(0)$ & $0.019^{\mathrm{b}}$ \\
Hypertension & $10(33.3)$ & $0(0)$ & $>0.999^{\mathrm{b}}$ \\
Diabetes & $1(0.3)$ & $0(0)$ & $0.545^{\mathrm{b}}$ \\
Coronary artery disease & $2(0.7)$ & $0(0)$ & $0.545^{\mathrm{b}}$ \\
Hyperlipidemia & $2(0.7)$ & $2(13.3)$ & $0.456^{\mathrm{b}}$ \\
History of smoking & $8(26.7)$ & $31.8 \pm 2.2$ & $<0.001^{\mathrm{a}}$ \\
Ascending aortic diameter $(\mathrm{mm})$ & $43.9 \pm 6.9$ & $0(0)$ & $<0.001^{\mathrm{b}}$ \\
Ascending aortic diameter $\geq 45 \mathrm{~mm}$ & $17(56.7)$ & & $<0.001^{\mathrm{c}}$ \\
Severity of aortic stenosis & & $0(0)$ & $0(0)$
\end{tabular}

Continuous variables are expressed as means \pm standard deviations, and categorical variables as numbers (percentages). Data are compared using ${ }^{\text {aS }}$ tudent's t-test, ${ }^{\mathrm{b}}$ Fisher's exact test, and 'Cochran-Mantel-Haenszel test. BAV, bicuspid aortic valve.

Table S2 Baseline characteristics of the patients from whom the aortic smooth muscle cells were harvested

\begin{tabular}{|c|c|c|c|c|c|}
\hline Patients & Age (year) & Gender & Hypertension & Ascending aortic diameter $(\mathrm{mm})$ & Aortic stenosis \\
\hline Control-2 & 22 & Male & No & N/A & No \\
\hline BAV-1 & 50 & Female & No & 43 & Severe \\
\hline BAV-2 & 46 & Male & Yes & 53 & Severe \\
\hline
\end{tabular}

BAV, bicuspid aortic valve. 


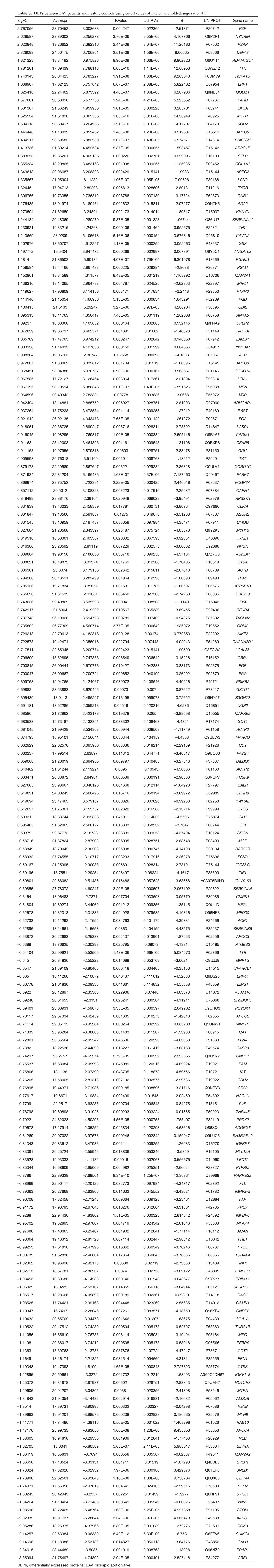




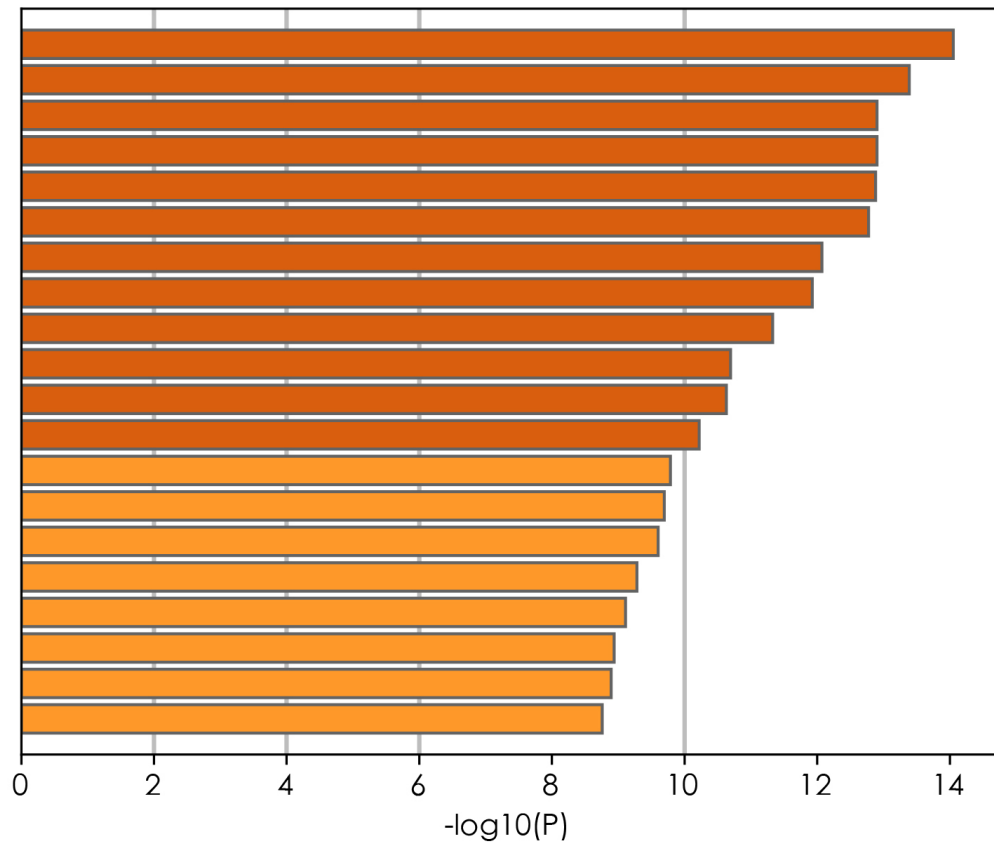

Myocardial Ischemia

Vascular Diseases

Hepatitis, Toxic

Chemically-Induced Liver Toxicity

Drug-Induced Acute Liver Injury

Hepatitis, Drug-Induced

Lupus Nephritis

Chemical and Drug Induced Liver Injury

Endothelial dysfunction

Down Syndrome

Drug-Induced Liver Disease

IGA Glomerulonephritis

Mild cognitive disorder

Creutzfeldt-Jakob disease

Complete Trisomy 21 Syndrome

Amyloidosis

Aortic Aneurysm, Abdominal

Forgetful

Undifferentiated carcinoma

Keloid

Figure S1 Disease enrichment analysis of the DEPs between BAV patients and controls. Cardiovascular diseases such as vascular diseases and abdominal aortic aneurysm (labeled red) are highly enriched. DEPs, differentially expressed proteins; BAV, bicuspid aortic valve.

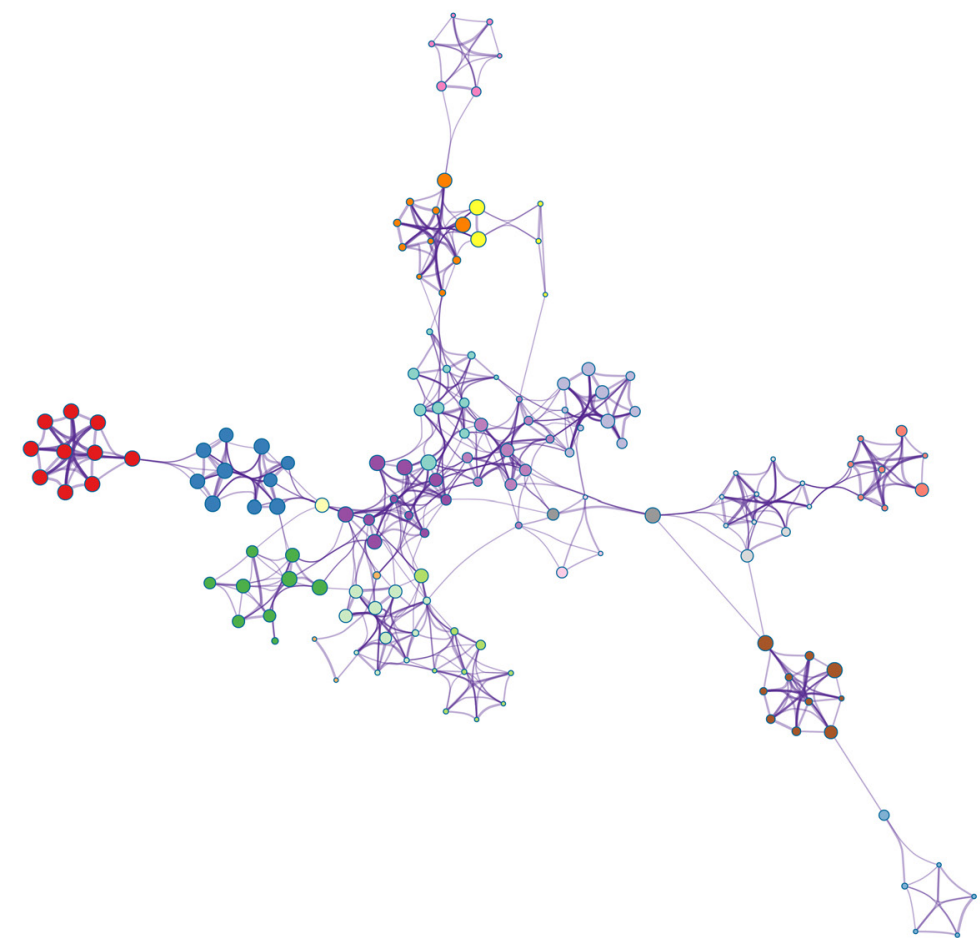

regulated exocytosis

Platelet degranulation

extracellular structure organization

cell morphogenesis involved in differentiation

Arp $2 / 3$ protein complex

Vesicle-mediated transport

Metabolism of carbohydrates

Phagosome

response to inorganic substance

positive regulation of cell development

VEGFA-VEGFR2 Signaling Pathway

regulation of cation transmembrane transport

Interleukin-12 family signaling

Diseases of metabolism

Human Complement System

regulation of vesicle-mediated transport

maintenance of location

response to oxidative stress

regulation of cellular protein localization

positive regulation of cell migration

Figure S2 The top 20 clusters of the enriched biological processes of the DEPs between BAV patients and control. DEPs, differentially expressed proteins; BAV, bicuspid aortic valve. 


\begin{tabular}{|c|c|c|c|c|}
\hline$P$ value & $\log F \mathrm{C}$ & Adjust $P$ value & UNIPROT & Gene name \\
\hline 0.023843 & -0.78052 & 0.375216 & A0A075B6K4 & IGLV3-10 \\
\hline 0.007486 & -1.77323 & 0.294705 & A0A0J9YXX1 & IGHV5-10-1 \\
\hline 0.015418 & 0.391122 & 0.365986 & 000533 & CHL1 \\
\hline 0.017985 & -1.40035 & 0.365986 & 014672 & ADAM10 \\
\hline 0.005385 & -2.06706 & 0.260209 & 043488 & AKRTA2 \\
\hline 0.002163 & 1.22667 & 0.257634 & 043493 & TGOLN2 \\
\hline 0.010086 & 1.664424 & 0.328 & 043852 & CALU \\
\hline 0.018944 & 1.0609 & 0.366444 & 076074 & PDE5A \\
\hline 0.03157 & -0.57577 & 0.390214 & P00441 & SOD1 \\
\hline 0.020188 & -0.54302 & 0.366444 & P00533 & EGFR \\
\hline 0.049681 & -0.32422 & 0.502177 & P00568 & $A K 1$ \\
\hline 0.005015 & -0.58994 & 0.260209 & P00740 & $F 9$ \\
\hline 0.00932 & -0.19366 & 0.316878 & P00742 & $F 10$ \\
\hline 0.000971 & 0.304493 & 0.242217 & P01008 & SERPINC1 \\
\hline 0.021269 & -0.37487 & 0.366444 & P01031 & C5 \\
\hline 0.029746 & -1.04776 & 0.390214 & P01876 & IGHA1 \\
\hline 0.03172 & -0.8792 & 0.390214 & P02655 & $A P O C 2$ \\
\hline 0.002227 & -2.45432 & 0.257634 & P02741 & CRP \\
\hline 0.035287 & -0.42657 & 0.410234 & P02753 & RBP4 \\
\hline 0.000256 & 0.508344 & 0.191618 & P05090 & $A P O D$ \\
\hline 0.028416 & -2.59689 & 0.390214 & P05121 & SERPINE1 \\
\hline 0.005419 & -0.36726 & 0.260209 & P05156 & CFI \\
\hline 0.017291 & -0.48839 & 0.365986 & P07357 & $C 8 A$ \\
\hline 0.005568 & 1.556691 & 0.260209 & P07911 & UMOD \\
\hline 0.026802 & 0.436539 & 0.384033 & P08185 & SERPINA6 \\
\hline 0.000635 & -3.24178 & 0.237391 & P09972 & $A L D O C$ \\
\hline 0.026296 & -0.56556 & 0.384033 & POCOL4 & C4A \\
\hline 0.017998 & 1.462011 & 0.365986 & P10321 & HLA-C \\
\hline 0.02168 & -1.16282 & 0.366444 & P10644 & PRKAR1A \\
\hline 0.0031 & 0.686498 & 0.257634 & P10646 & TFPI \\
\hline 0.036714 & -2.05085 & 0.410234 & P13224 & GP1BB \\
\hline 0.033175 & 0.678009 & 0.400237 & P13716 & $A L A D$ \\
\hline 0.020199 & 0.772203 & 0.366444 & P16112 & $A C A N$ \\
\hline 0.036097 & -1.69914 & 0.410234 & P20023 & CR2 \\
\hline 0.04191 & 0.774869 & 0.454324 & P41240 & CSK \\
\hline 0.029134 & -1.58522 & 0.390214 & P48426 & PIP4K2A \\
\hline 0.027211 & -0.56463 & 0.384033 & P48637 & GSS \\
\hline 0.02432 & -1.90319 & 0.375216 & P49407 & ARRB1 \\
\hline 0.011427 & -0.47361 & 0.356128 & P51149 & RAB7A \\
\hline 0.014439 & 0.561058 & 0.365986 & P51884 & LUM \\
\hline 0.008061 & 0.913406 & 0.301471 & P54802 & NAGLU \\
\hline 0.008881 & -1.92216 & 0.316316 & P54819 & AK2 \\
\hline 0.013363 & -1.41434 & 0.365986 & P55056 & APOC4 \\
\hline 0.018104 & -1.13536 & 0.365986 & P61088 & UBE2N \\
\hline 0.044376 & -0.638 & 0.474189 & P61916 & NPC2 \\
\hline 0.046453 & -0.86744 & 0.482101 & P62942 & FKBP1A \\
\hline 0.006589 & -0.30224 & 0.273802 & P62979 & RPS27A \\
\hline 0.013144 & 0.63441 & 0.365986 & Q12860 & CNTN1 \\
\hline 0.005392 & -1.72557 & 0.260209 & Q14141 & SEPTIN6 \\
\hline 0.025081 & -1.45493 & 0.375216 & Q15019 & SEPTIN2 \\
\hline 0.020735 & 0.698228 & 0.366444 & Q15166 & PON3 \\
\hline 0.037294 & 0.884489 & 0.410234 & Q16706 & MAN2A1 \\
\hline 0.037136 & -2.90432 & 0.410234 & Q4LDE5 & SVEP1 \\
\hline 0.02485 & -1.22303 & 0.375216 & Q6EEV6 & SUMO4 \\
\hline 0.005914 & 1.008333 & 0.260209 & Q76LX8 & ADAMTS13 \\
\hline 0.030199 & 0.852641 & 0.390214 & Q7L591 & DOK3 \\
\hline 0.04705 & 0.741053 & 0.482101 & Q86TH1 & ADAMTSL2 2 \\
\hline 0.046495 & -0.89383 & 0.482101 & Q8NBJ4 & GOLM1 \\
\hline 0.02245 & 0.743088 & 0.366444 & Q92820 & GGH \\
\hline 0.014649 & -0.94753 & 0.365986 & Q96HR3 & MED30 \\
\hline 0.017085 & 1.489395 & 0.365986 & Q96NZ9 & PRAP1 \\
\hline 0.015545 & -3.026 & 0.365986 & Q99439 & CNN2 \\
\hline 0.030678 & -2.25868 & 0.390214 & Q99685 & MGLL \\
\hline 0.003553 & -1.2312 & 0.257634 & Q9BR76 & CORO1B \\
\hline 0.013768 & -1.65934 & 0.365986 & Q9BS26 & ERP44 \\
\hline 0.003369 & -0.38306 & 0.257634 & Q9BXR6 & CFHR5 \\
\hline 0.016244 & -2.33835 & 0.365986 & Q9HBB8 & CDHR5 \\
\hline 0.022535 & -1.10941 & 0.366444 & Q9HDC9 & APMAP \\
\hline 0.034891 & -1.40687 & 0.410234 & Q9NZ08 & ERAP1 \\
\hline 0.020586 & 0.870296 & 0.366444 & Q9UBQ6 & EXTL2 \\
\hline 0.001567 & -1.17653 & 0.257634 & Q9UJC5 & SH3BGRL2 \\
\hline 0.002935 & -2.53814 & 0.257634 & Q9UJU6 & DBNL \\
\hline 0.031822 & -1.11919 & 0.390214 & Q9UQ80 & PA2G4 \\
\hline 0.003789 & -2.23932 & 0.257634 & Q9Y696 & CLIC4 \\
\hline
\end{tabular}

